\title{
Forward flight stability in a drone-fly
}

\begin{abstract}
Hao Jie Zhu ${ }^{1 *}$, Xue Guang Meng ${ }^{2} \&$ Mao Sun ${ }^{1}$
Previous studies on forward flight stability in insects are for low to medium flight-speeds. In the present work, we investigated the stability problem for the full range of flight speeds $(0-8.6 \mathrm{~m} / \mathrm{s})$ of a drone-fly. Our results show the following: The longitudinal derivatives due to the lateral motion are approximately 3 orders of magnitude smaller than the other longitudinal derivatives. Thus, we can decouple these two motions of the insect, as commonly done for a conventional airplane. At hovering flight, the motion of the dronefly is weakly unstable owing to two unstable natural modes of motion, a longitudinal one and a lateral one. At low $(1.6 \mathrm{~m} / \mathrm{s})$ and medium $(3.1 \mathrm{~m} / \mathrm{s})$ flight-speeds, the unstable modes become even weaker and the flight is approximately neutral. At high flight-speeds $(4.6 \mathrm{~m} / \mathrm{s}, 6.9 \mathrm{~m} / \mathrm{s}$ and $8.6 \mathrm{~m} / \mathrm{s})$, the flight becomes more and more unstable due to an unstable longitudinal mode. At the highest flight speed, $8.6 \mathrm{~m} / \mathrm{s}$, the instability is so strong that the time constant representing the growth rate of the instability (disturbance-doubling time) is only $10.1 \mathrm{~ms}$, which is close to the sensory reaction time of a fly (approximately $11 \mathrm{~ms}$ ). This indicates that strong instability may play a role in limiting the flight speed of the insect.
\end{abstract}

When the lift balances the weight, the thrust balances the drag and the moments about the center of mass are zero, an insect is in equilibrium flight. Dynamic stability answers the question that when the flight is disturbed, whether or not the insect returns to its equilibrium state without applying active control. Knowing whether or not the flight is stable and if it is unstable, how fast the instability grows, is essential to understanding the controllability and maneuverability of the insect. It also is of great importance to the study of its internal control systems, e.g. sensory systems and motor systems. Besides, understanding the flight stability of insects can be helpful in designing insect-like flying robots. As a result, in the last fifteen years or so, many studies have been conducted in this area ${ }^{1-14}$. However, most of these previous works were for hovering flight, while very few were on forward flight. As far as we know, only three studies were on forward flight: Taylor and Thomas ${ }^{1}$ investigated the stability in forward-flying locusts at medium flight speed. Only longitudinal motion was considered. In the study, the aerodynamic derivatives were measured and the insect was tethered in the experiment. They obtained an unstable divergence mode and two stable modes (a subsidence one and an oscillatory one, respectively). Xiong and $\operatorname{Sun}^{13}$ investigated the longitudinal flight dynamics in forward flight in a bumblebee. Xu and Sun ${ }^{14}$ consid- $^{-}$ ered the lateral flight dynamics of the same insect. Flight dynamics at hover and at speeds $1 \mathrm{~m} / \mathrm{s}, 2.5 \mathrm{~m} / \mathrm{s}, 3.5 \mathrm{~m} / \mathrm{s}$ and $4.5 \mathrm{~m} / \mathrm{s}$ was studied. Natural modes of motion at each flight speed were identified. Morphologic parameters and flight data of the insect were taken from literature ${ }^{15}$. Hovering flight and low speed flight have similar model structure: three longitudinal modes, one of them being a weakly unstable oscillatory mode and the same number of lateral modes, one of them being weakly unstable. The flight is unstable owing to the two modes of instability. As speed increases, the model structure changes and the flight is approximately neutrally stable at speeds $2.5 \mathrm{~m} / \mathrm{s}$ and $3.5 \mathrm{~m} / \mathrm{s}$ and becomes unstable again at speed $4.5 \mathrm{~m} / \mathrm{s}$. The instability at speed $4.5 \mathrm{~m} / \mathrm{s}$ is stronger than that hovering flight.

In Taylor and Thomas' study, aerodynamic derivatives were measured from a "flying" locust ${ }^{1}$. In this type of experiment, control responses of the insect must have been introduced to the measurement and inherent (or passive) stability derivatives cannot be obtained due to the tethering. Thus, the results they obtained may not represent the passive stability of the insect. In the bumblebee study of Xiong and Sun ${ }^{13}$ and Xu and Sun ${ }^{14}$, passive stability derivatives were obtained by computation; but the flight-speed range was limited to $0-4.5 \mathrm{~m} / \mathrm{s}$, because flight data were only measured in this speed range ${ }^{15}$. As described above, at velocity of $4.5 \mathrm{~m} / \mathrm{s}$, the motion is more unstable. What the flight stability properties would become at higher speeds is unknown. Stability properties for the full range of speed of an insect are of great interest.

Recently, our group measured the flapping kinematics of freely-flying droneflies ${ }^{16}$. The speed range is $0-8.6 \mathrm{~m} / \mathrm{s}$. It was shown that the insect could not fly at speeds higher than about $8.6 \mathrm{~m} / \mathrm{s}$ and therefore the above flight speed range could represent the full speed range of the insect. The reason for choosing droneflies for the

${ }^{1}$ Ministry-of-Education Key Laboratory of Fluid Mechanics, School of Aeronautic and Engineering, Beihang University, Beijing, 100191, China. ${ }^{2}$ State Key Laboratory for Strength and Vibration of Mechanical Structures, School of Aerospace, Xi'an Jiaotong University, Xi'an, 710049, China. *email: by1505144@buaa.edu.cn 


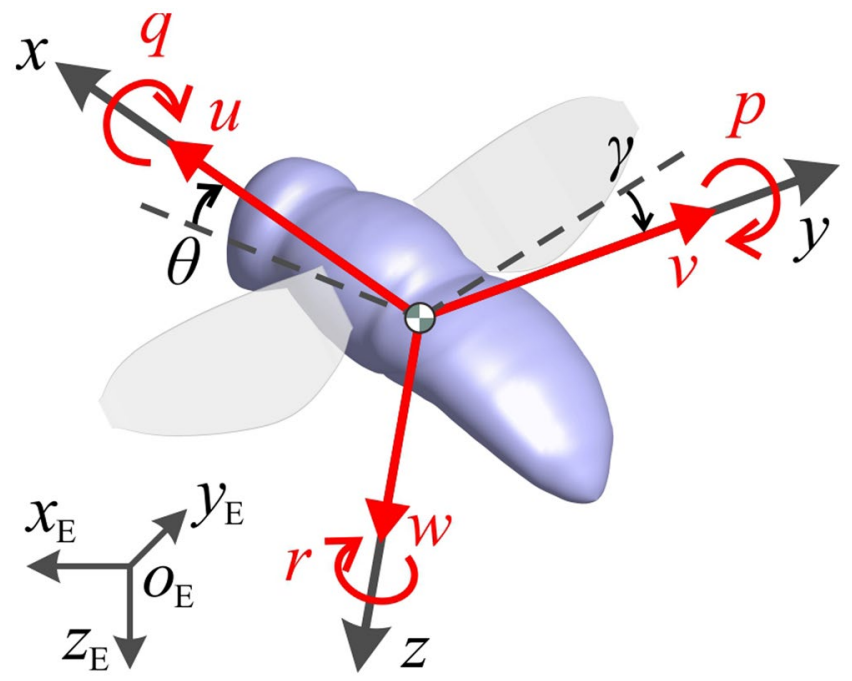

Figure 1. A sketch showing the reference frames and state variables. $x y z$ is a non-inertial frame fixed on the body and $x_{\mathrm{E}} y_{\mathrm{E}} z_{\mathrm{E}}$ is a laboratory frame. The origin of the $x y z$ frame is at the center of mass of the insect.

experiment was that they fly very well in the experiment conditions. And moreover, the size of dronefly is approximately the same as that of the medium-sized bumblebee and results can therefore be compared between the two. Here, we investigate the stability properties of a forward-flying dronefly in the full speed range of the insect $(0-8.6 \mathrm{~m} / \mathrm{s})$, using the above mentioned measured data, including hover flight. With this study, we can answer the above question that at higher flight speed, the flight will become more unstable or less unstable.

\section{Materials and Methods}

As in our previous works in this area ${ }^{2,13,14}$, averaged-model (see below) is used to represent the dynamics of the insect and linearization theory is applied to the problem. We solve the Navier-Stokes equations numerically to obtain the aerodynamic derivatives, so that inherent (or passive) stability derivative can be obtained. At each flight speed, first, aerodynamic forces and moments at balanced flight are calculated using the measured flapping kinematics from Meng and Sun ${ }^{16}$. Then we compute the aerodynamic derivatives. Finally, we analize the stability properties at each flight speed by examining the natural modes of motion.

Reference frames and equations of motion. A flying insect is a nonlinear time-variant dynamic system. For such a system, the oscillating mass distribution and the periodic aerodynamic and inertial forces associated with the oscillating wings can couple with the body's natural modes of motion. But if the wingbeat frequency is sufficiently high compared to the insect's natural frequencies of gross motion, such a coupling is unlikely to occur. In this case, one may consider replacing the forces by wingbeat-cycle average forces, which might vary over the time scale of the gross motion of the insect. This is referred to as averaged-model. Detailed description of the averaged-model can be found in Taylor and Thomas ${ }^{1}$ and Sun and Xiong ${ }^{2}$. In the averaged-model, the insect is represented by rigid body (Fig. 1); the aerodynamic forces and moments of the wing are time-averaged over each flapping period; these mean forces and moments vary with time during the disturbed motion.

Thus the equations of motion is similar to those of an airplane or helicopter, which can be found in Etkin and Reid's book on flight dynamics of airplanes ${ }^{17}$ (or any other textbook on this subject):

$$
\begin{gathered}
X-m g \sin \theta=m(\dot{u}+w q-v r) \\
Y+m g \cos \theta \sin \gamma=m(\dot{v}+r u-p w) \\
Z+m g \cos \theta \cos \gamma=m(\dot{w}-q u+p v) \\
L=I_{x} \dot{p}-I_{x z} \dot{r}-I_{x z} p q-\left(I_{y}-I_{z}\right) q r \\
M=I_{y} \dot{q}+I_{x z}\left(p^{2}-r^{2}\right)+\left(I_{x}-I_{z}\right) r q \\
N=-I_{x z} \dot{p}+I_{z} \dot{r}-\left(I_{x}-I_{y}\right) p q+I_{x z} q r \\
\dot{\gamma}=p+\tan \theta(q \sin \gamma+r \cos \gamma)
\end{gathered}
$$




$$
\begin{gathered}
\dot{\theta}=q \cos \gamma-r \sin \gamma \\
\dot{\psi}=\frac{1}{\cos \theta}(q \sin \gamma+r \cos \gamma)
\end{gathered}
$$

where $u$ is the $x$-component of the velocity of the center of mass (Fig. 1), $v$ and $w$ are the corresponding $y$ and $z$ components, respectively; $\theta$ is the pitch angle, $\gamma$ is the roll angle and $\psi$ is yaw angle (Fig. 1$)(u, v, w, \theta, \gamma$ and $\psi$ are referred to as state variables); $X$ is the $x$-component of the aerodynamic force acting on the insect and $Y$ and $Z$ are the corresponding $y$-and $z$-components, respectively; $L$ is the $x$-component of the aerodynamic moment, and $Y$ and $Z$ are the corresponding $y$-and $z$-components, respectively (note that they are contributed by both the wings and the body); $m$ is insect mass; $g$ is the gravity constant; $I_{\mathrm{x}}, I_{\mathrm{y}}$ and $I_{\mathrm{z}}$ are the moments of inertia about the $x$-, $y$ - and $z$-axis, respectively, and $I_{\mathrm{xz}}$ is corresponding product of inertia; "." indicates taking time $(t)$ derivative. We can see that Eq. (9) is decoupled from Eqs. (1)-(8) since the yaw angle $(\psi)$ does not appear in Eqs. (1)-(8).

Although Eqs. (1)-(8) are non-linear, but they can be linearized by assuming the disturbance motion from the equilibrium flight is small, ${ }^{1,2}$ That is, we write $u=u_{\mathrm{e}}+\delta u, v=v_{\mathrm{e}}+\delta v$ etc., where the subscript "e" denotes the equilibrium-flight condition and the symbol " $\delta$ " indicates a small quantity; we also write $X=X_{\mathrm{e}}+\delta X, Y=Y_{\mathrm{e}}+\delta Y$ etc. The six force and moment disturbances, $\delta X, \delta Y$, etc. are further expanded as Taylor's series in terms of the disturbance state variables (the nonlinear terms are dropped) ${ }^{1,2}$ :

$$
\begin{gathered}
\delta X=X_{\mathrm{u}} \delta u+X_{\mathrm{v}} \delta v+X_{\mathrm{w}} \delta w+X_{\mathrm{p}} \delta p+X_{\mathrm{q}} \delta q+X_{\mathrm{r}} \delta r \\
\delta Y=Y_{\mathrm{u}} \delta u+Y_{\mathrm{v}} \delta v+Y_{\mathrm{w}} \delta w+Y_{\mathrm{p}} \delta p+Y_{\mathrm{q}} \delta q+Y_{\mathrm{r}} \delta r \\
\delta Z=Z_{\mathrm{u}} \delta u+Z_{\mathrm{v}} \delta v+Z_{\mathrm{w}} \delta w+Z_{\mathrm{p}} \delta p+Z_{\mathrm{q}} \delta q+Z_{\mathrm{r}} \delta r \\
\delta L=L_{\mathrm{u}} \delta u+L_{\mathrm{v}} \delta v+L_{\mathrm{w}} \delta w+L_{\mathrm{p}} \delta p+L_{\mathrm{q}} \delta q+L_{\mathrm{r}} \delta r \\
\delta M=M_{\mathrm{u}} \delta u+M_{\mathrm{v}} \delta v+M_{\mathrm{w}} \delta w+M_{\mathrm{p}} \delta p+M_{\mathrm{q}} \delta q+M_{\mathrm{r}} \delta r \\
\delta N=N_{\mathrm{u}} \delta u+N_{\mathrm{v}} \delta v+N_{\mathrm{w}} \delta w+N_{\mathrm{p}} \delta p+N_{\mathrm{q}} \delta q+N_{\mathrm{r}} \delta r
\end{gathered}
$$

where $X_{\mathrm{u}}, X_{\mathrm{v}}$, etc. are the aerodynamic or stability derivatives. Since the insect is symmetrical with respect to the vertical plane passing the longitudinal axis, the longitudinal disturbance motion $(\delta u, \delta w, \delta q$ and $\delta \theta)$ will not produce lateral aerodynamic forces and moments, thus the lateral aerodynamic derivatives due to longitudinal disturbance motion are zero, i.e., $Y_{\mathrm{u}}=Y_{\mathrm{w}}=Y_{\mathrm{q}}=L_{\mathrm{u}}=L_{\mathrm{w}}=L_{\mathrm{q}}=N_{\mathrm{u}}=N_{\mathrm{w}}=N_{\mathrm{q}}=0$. Therefore, Eqs. (10)-(15) can be simplified as:

$$
\begin{gathered}
\delta X=X_{\mathrm{u}} \delta u+X_{\mathrm{v}} \delta v+X_{\mathrm{w}} \delta w+X_{\mathrm{p}} \delta p+X_{\mathrm{q}} \delta q+X_{\mathrm{r}} \delta r \\
\delta Y=Y_{\mathrm{v}} \delta v+Y_{\mathrm{p}} \delta p+Y_{\mathrm{r}} \delta r \\
\delta Z=Z_{\mathrm{u}} \delta u+Z_{\mathrm{v}} \delta v+Z_{\mathrm{w}} \delta w+Z_{\mathrm{p}} \delta p+Z_{\mathrm{q}} \delta q+Z_{\mathrm{r}} \delta r \\
\delta L=L_{\mathrm{v}} \delta v+L_{\mathrm{p}} \delta p+L_{\mathrm{r}} \delta r \\
\delta M=M_{\mathrm{u}} \delta u+M_{\mathrm{v}} \delta v+M_{\mathrm{w}} \delta w+M_{\mathrm{p}} \delta p+M_{\mathrm{q}} \delta q+M_{\mathrm{r}} \delta r \\
\delta N=N_{\mathrm{v}} \delta v+N_{\mathrm{p}} \delta p+N_{\mathrm{r}} \delta r
\end{gathered}
$$

For a conventional airplane, it has been shown that the lateral disturbance motion $(\delta v, \delta p, \delta r$ and $\delta \gamma)$ will only produce negligibly small longitudinal disturbance ${ }^{17}$. Therefore, the longitudinal aerodynamic derivatives due to lateral disturbance motion are approximately zero, i.e.

$$
X_{\mathrm{v}} \approx 0, Z_{\mathrm{v}} \approx 0, M_{\mathrm{v}} \approx 0, X_{\mathrm{p}} \approx 0, Z_{\mathrm{p}} \approx 0, M_{\mathrm{p}} \approx 0, X_{\mathrm{r}} \approx 0, Z_{\mathrm{r}} \approx 0, M_{\mathrm{r}} \approx 0
$$

and Eqs. (16)-(21) can be further simplified. For an insect in flapping flight, especially in forward flight, whether or not this is true is unknown. We will examine this in our calculation below.

At reference (or equilibrium) flight, $u_{\mathrm{e}}=V_{\mathrm{e}}\left(V_{\mathrm{e}}\right.$ is the forward flight speed) and $v_{\mathrm{e}}, w_{\mathrm{e}}, p_{\mathrm{e}}, q_{\mathrm{e}}, r_{\mathrm{e}}, \theta_{\mathrm{e}}, \gamma_{\mathrm{e}}$ are zero (the zero $\theta_{\mathrm{e}}$ and $\gamma_{\mathrm{e}}$ are due to the $x$-y plane being horizontal at equilibrium flight); $Z_{\mathrm{e}}=-m g$ (vertcal forces balance te weight); $X_{\mathrm{e}}, Y_{\mathrm{e}}, L_{\mathrm{e}}, M_{\mathrm{e}}, N_{\mathrm{e}}$ are zero (the insect is in equilibrium state). Substituting the expressions of independent state variables and Eqs. (16)-(21) into Eqs. (1)-(8), the linearized equations of motion can be obtained and they are expressed in the following non-dimensional matrix form: 


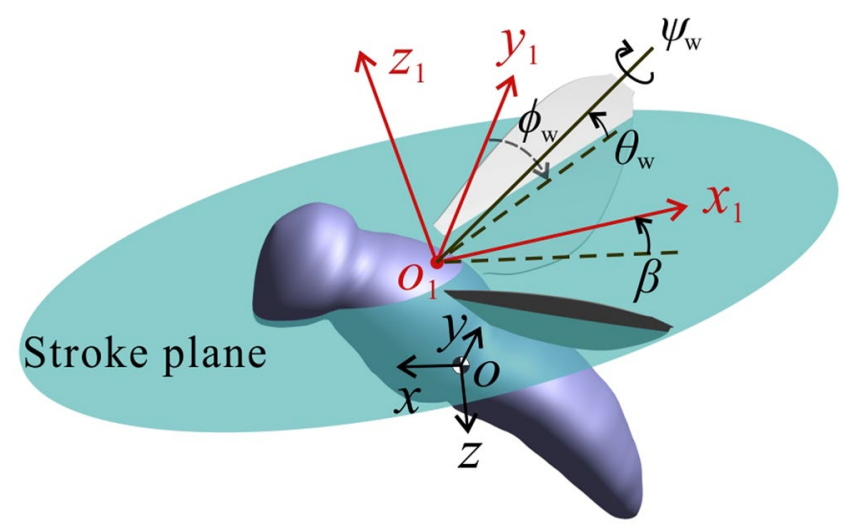

Figure 2. Definitions of the wing kinematics.

$$
\left[\begin{array}{c}
\delta \dot{u}^{+} \\
\delta \dot{w}^{+} \\
\delta \dot{q}^{+} \\
\delta \dot{\theta}^{+} \\
\delta \dot{v}^{+} \\
\delta \dot{p}^{+} \\
\delta \dot{r}^{+} \\
\delta \dot{\gamma}^{+}
\end{array}\right]=\mathbf{A}\left[\begin{array}{l}
\delta u^{+} \\
\delta w^{+} \\
\delta q^{+} \\
\delta \theta^{+} \\
\delta v^{+} \\
\delta p^{+} \\
\delta r^{+} \\
\delta \gamma^{+}
\end{array}\right]
$$

where $\mathbf{A}$ is referred to as system matrix:

$$
\mathbf{A}=\left[\begin{array}{cccccccc}
\frac{X_{\mathrm{u}}^{+}}{m^{+}} & \frac{X_{\mathrm{w}}^{+}}{m^{+}} & \frac{X_{\mathrm{q}}^{+}}{m^{+}} & -g^{+} & \frac{X_{\mathrm{v}}^{+}}{m^{+}} & \frac{X_{\mathrm{p}}^{+}}{m^{+}} & \frac{X_{\mathrm{r}}^{+}}{m^{+}} & 0 \\
\frac{Z_{\mathrm{u}}^{+}}{m^{+}} & \frac{Z_{\mathrm{w}}^{+}}{m^{+}} & \frac{Z_{\mathrm{q}}^{+}}{m^{+}}+V_{\mathrm{e}}^{+} & 0 & \frac{Z_{\mathrm{v}}^{+}}{m^{+}} & \frac{Z_{\mathrm{p}}^{+}}{m^{+}} & \frac{Z_{\mathrm{r}}^{+}}{m^{+}} & 0 \\
\frac{M_{\mathrm{u}}^{+}}{I_{\mathrm{y}}^{+}} & \frac{M_{\mathrm{w}}^{+}}{I_{\mathrm{y}}^{+}} & \frac{M_{\mathrm{q}}^{+}}{I_{\mathrm{y}}^{+}} & 0 & \frac{M_{\mathrm{v}}^{+}}{I_{\mathrm{y}}^{+}} & \frac{M_{\mathrm{p}}^{+}}{I_{\mathrm{y}}^{+}} & \frac{M_{\mathrm{r}}^{+}}{I_{\mathrm{y}}^{+}} & 0 \\
0 & 0 & 1 & 0 & 0 & 0 & 0 & 0 \\
0 & 0 & 0 & 0 & \frac{Y_{\mathrm{v}}^{+}}{m^{+}} & \frac{Y_{\mathrm{p}}^{+}}{m^{+}} & \frac{Y_{\mathrm{r}}^{+}}{m^{+}}-V_{\mathrm{e}}^{+} & g^{+} \\
0 & 0 & 0 & 0 & \frac{I_{\mathrm{z}}^{+} L_{\mathrm{v}}^{+}+I_{\mathrm{xz}}^{+} N_{\mathrm{v}}^{+}}{I_{\mathrm{x}}^{+} I_{\mathrm{z}}^{+}-I_{\mathrm{xz}}^{+2}} & \frac{I_{\mathrm{z}}^{+} L_{\mathrm{p}}^{+}+I_{\mathrm{xz}}^{+} N_{\mathrm{p}}^{+}}{I_{\mathrm{x}}^{+} I_{\mathrm{z}}^{+}-I_{\mathrm{xz}}^{+2}} \frac{I_{\mathrm{z}}^{+} L_{\mathrm{r}}^{+}+I_{\mathrm{xz}}^{+} N_{\mathrm{r}}^{+}}{I_{\mathrm{x}}^{+} I_{\mathrm{z}}^{+}-I_{\mathrm{xz}}^{+2}} & 0 \\
0 & 0 & 0 & 0 & \frac{I_{\mathrm{xz}}^{+} L_{\mathrm{v}}^{+}+I_{\mathrm{x}}^{+} N_{\mathrm{v}}^{+}}{I_{\mathrm{x}}^{+} I_{\mathrm{z}}^{+}-I_{\mathrm{xz}}^{+2}} & \frac{I_{\mathrm{xz}}^{+} L_{\mathrm{p}}^{+}+I_{\mathrm{x}}^{+} N_{\mathrm{p}}^{+}}{I_{\mathrm{x}}^{+} I_{\mathrm{z}}^{+}-I_{\mathrm{xz}}^{+2}} & \frac{I_{\mathrm{xz}}^{+} L_{\mathrm{r}}^{+}+I_{\mathrm{x}}^{+} N_{\mathrm{r}}^{+}}{I_{\mathrm{x}}^{+} I_{\mathrm{z}}^{+}-I_{\mathrm{xz}}^{+2}} & 0 \\
0 & 0 & 0 & 0 & 0 & 1 & 0 & 0
\end{array}\right]
$$

When non-dimensionalizing the equations, we take the mean wing-chord length $(c)$ as the reference length, the mean flapping velocity at hovering flight $(U)$ as the reference velocity (here $U=2 \Phi n r_{2} \Phi$ is the stroke amplitude at hovering flight, $r_{2}$ is the radius of the second moment of wing area and $n$ is the flapping frequency at hovering), and $T=1 / n$ as the reference time. The non-dimensional forms of the variables are: $V_{\mathrm{e}}^{+}=V_{\mathrm{e}} / U, \delta u^{+}=\delta u / U$, $\delta v^{+}=\delta v / U, \delta w^{+}=\delta w / U, \delta p^{+}=\delta p T, \delta q^{+}=\delta q T, \delta r^{+}=\delta r T, X^{+}=X / 0.5 \rho U^{2} S_{\mathrm{t}}$ (here $\rho$ is the air density; $S_{\mathrm{t}}$ is the area of the wing-pair), $Y^{+}=Y / 0.5 \rho U^{2} S_{\mathrm{t}}, Z^{+}=Z / 0.5 \rho U^{2} S_{\mathrm{t}}, L^{+}=L / 0.5 \rho U^{2} S_{\mathrm{t}} c, M^{+}=M / 0.5 \rho U^{2} S_{\mathrm{t}} c, N^{+}=N / 0.5 \rho U^{2} S_{\mathrm{t}} c$, $t^{+}=t / T, m^{+}=m / 0.5 \rho U S_{\mathrm{t}} T, I_{\mathrm{x}}^{+}=I_{\mathrm{x}} / 0.5 \rho U^{2} S_{\mathrm{t}} c T^{2}, I_{\mathrm{y}}{ }^{+}=I_{\mathrm{y}} / 0.5 \rho U^{2} S_{\mathrm{t}} c T^{2}, I_{\mathrm{z}}^{+}=I_{\mathrm{z}} / 0.5 \rho U^{2} S_{\mathrm{t}} c T^{2}, I_{\mathrm{xz}}{ }^{+}=I_{\mathrm{xz}} / 0.5 \rho U^{2} S_{\mathrm{t}} c T^{2}$ and $g^{+}=g T / U$.

Flight data. To solve Eqs. (23) and (24), we need aerodynamic derivatives, insect mass and insect moments of inertia and product of inertia. The mass and the moments and product of inertia are available from ref. ${ }^{16}$ or can be computed using data given in ref. ${ }^{16}$. The aerodynamic or stability derivatives are computed in the present study. Wing kinematics are needed for computing the aerodynamic derivatives. Wing kinematics of the dronefly is available in ref. ${ }^{16}$. For the wing-kinematics description, we follow Meng and Sun ${ }^{16}$ : The stroke plane and three flapping angles are defined as shown in Fig. 2. $o_{1} x_{1} y_{1} z_{1}$ is a coordinate system with $o_{1}$ at the wing-root and $x_{1}$ and $y_{1}$ in the stroke plane. The stroke plane angle is denoted as $\beta$ and it is the angle between the horizontal plane and the stroke plane when the insect is at equilibrium flight. The flapping angles include $\phi_{\mathrm{w}}, \theta_{\mathrm{w}}$ and $\psi_{\mathrm{w}}: \phi_{\mathrm{w}}$ is the 

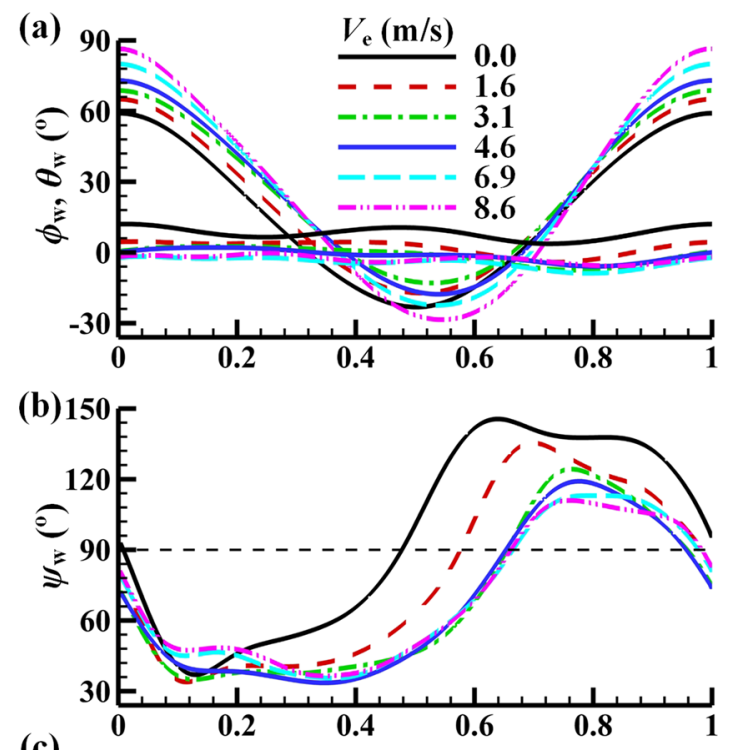

(c)

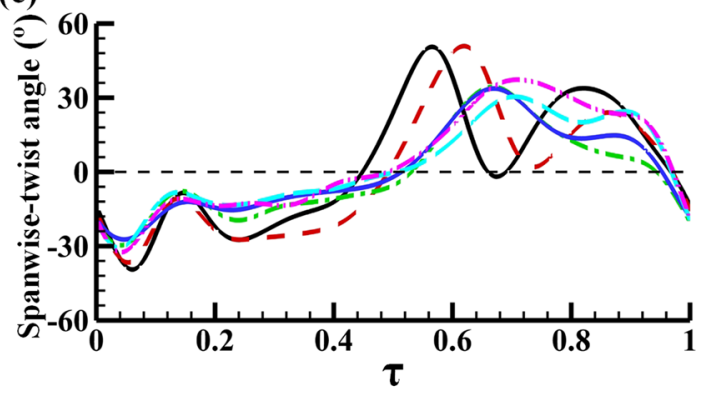

Figure 3. Wing motion of dronefly in a flapping period; data for six flight speeds. $\tau=0$ is the start of a downstroke; $\tau=1$ is the end of the following upstroke.

\begin{tabular}{|l|l|l|l|l|}
\hline $\boldsymbol{V}_{\mathbf{e}}(\mathbf{m} / \mathbf{s})$ & $\boldsymbol{n}(\mathbf{H z})$ & $\boldsymbol{\Phi}\left({ }^{\circ}\right)$ & $\boldsymbol{\beta}\left(^{\circ}\right)$ & $\chi\left(^{\circ}\right)$ \\
\hline 0.0 & 178 & 85 & 11 & 30 \\
\hline 1.6 & 166 & 83 & 19 & 24 \\
\hline 3.1 & 164 & 82 & 26 & 17 \\
\hline 4.6 & 167 & 92 & 37 & 7 \\
\hline 6.9 & 173 & 103 & 46 & 3 \\
\hline 8.6 & 180 & 115 & 50 & 1 \\
\hline
\end{tabular}

Table 1. Kinematic parameters of the dronefly.

positional angle, $\theta_{\mathrm{w}}$ is the stroke deviation angle, $\psi_{\mathrm{w}}$ is the pitch angle. The detailed description of $\phi_{\mathrm{w}}, \theta_{\mathrm{w}}$ and $\psi_{\mathrm{w}}$ can be found in Taylor and Thomas ${ }^{1}$ and Sun and Xiong ${ }^{2}$. With $\beta, \phi_{\mathrm{w}}, \theta_{\mathrm{w}}$ and $\psi_{\mathrm{w}}$ known, the wing-kinematics is determined. Body orientation is determined by the body angle $\chi$, which is the angle between body and the horizontal.

Values of the measured $\phi_{w}, \theta_{w}, \psi_{w}$ and spanwise-twist angle in one flapping cycle for the dronefly are shown in Fig. 3. The flapping amplitude, $\Phi$, is defined as the difference between the maximum $\phi_{\mathrm{w}}$ and the minimum $\phi_{\mathrm{w}^{*}}$. The values of $\Phi, n, \beta$ and $\chi$ are listed in Table 1 ; they are needed in the non-dimensionalization and in the computations.

The morphological parameters are: $m=151 \mathrm{mg}$; wing-length $R=13.05 \mathrm{~mm} ; c=3.31 \mathrm{~mm}, r_{2} / R=0.55$; single wing area $S=43.23 \mathrm{~mm}^{2}$; body length $l_{\mathrm{b}}=16.21 \mathrm{~mm}$; distance from head to center of mass divided by body length is $l_{2} / l_{\mathrm{b}}=0.48$; distance from wing base axis to center of mass divided by body length $l_{1} / l_{\mathrm{b}}=0.12$; distance between the two wing roots divided by body length $l_{\mathrm{r}} / l_{\mathrm{b}}=0.29$; the free body angle $\chi_{0}=59.5^{\circ} . I_{\mathrm{x}}, I_{\mathrm{y}}, I_{\mathrm{z}}$ and $I_{\mathrm{xz}}$ are calculated using the flight data given in Meng and Sun ${ }^{16}$, using the method described in our previous papers ${ }^{14,18}$. Note that at different flight speed the body position relative to the oxyz coordinate frame is different, therefore, $I_{\mathrm{x}}, I_{\mathrm{y}}, I_{\mathrm{z}}$ and $I_{\mathrm{xz}}$ are different for different flight speed; the values are given in Table 2. 


\begin{tabular}{|l|l|l|l|l|}
\hline$V_{\mathrm{e}}(\mathbf{m} / \mathbf{s})$ & $\boldsymbol{I}_{\mathbf{x}}\left(\mathbf{m g} \cdot \mathbf{m m}^{2}\right)$ & $\boldsymbol{I}_{\mathbf{y}}\left(\mathbf{m g} \cdot \mathbf{m m}^{2}\right)$ & $\boldsymbol{I}_{\mathbf{z}}\left(\mathbf{m g} \cdot \mathbf{m m}^{2}\right)$ & $\boldsymbol{I}_{\mathbf{x z}}\left(\mathbf{m g} \cdot \mathbf{m m}^{2}\right)$ \\
\hline 0.0 & 987.1 & 2699.7 & 2379.1 & 971.7 \\
\hline 1.6 & 796.6 & 2698.9 & 2559.5 & 804.4 \\
\hline 3.1 & 625.2 & 2698.9 & 2723.5 & 566.7 \\
\hline 4.6 & 494.9 & 2698.9 & 2853.7 & 173.7 \\
\hline 6.9 & 482.8 & 2699.0 & 2863.9 & 7.9 \\
\hline 8.6 & 485.7 & 2699.0 & 2861.0 & -75.0 \\
\hline
\end{tabular}

Table 2. The moments of inertia and the products of inertia.

Calculation of the aerodynamic derivatives. The aerodynamic derivatives are calculated by solution of the flow equations (the Navier-Stokes equations). Moving overset grids are employed owing to the relative motion of the wings and body. The flow solver is the same as that employed in several previous works of our group ${ }^{14,19,20}$ and the description of the method is presented in the Supplementary Material, Supplementary S1. Tests of the computational grid are also given in the Supplementary Material, Supplementary S2.

Solving the equations of motion. After the morphological parameters and flight data are given and the aerodynamic derivatives are calculated, the matrixes $\mathbf{A}$ in Eq. (23) are determined. We are ready to solve Eq. (23) and analyze the flight stability of the dronefly.

Let $\boldsymbol{x}$ be the state variable vector in Eq. (23):

$$
\boldsymbol{x}=\left[\begin{array}{l}
\delta u \\
\delta w \\
\delta q \\
\delta \theta \\
\delta v \\
\delta p \\
\delta r \\
\delta \gamma
\end{array}\right]
$$

The solution of Eq. (23) is ${ }^{17}$ :

$$
\boldsymbol{x}=\sum_{i=1}^{8} \boldsymbol{a}_{\mathrm{i}} e^{\lambda_{\mathrm{i}} t^{+}}
$$

where $\lambda_{\mathrm{i}}$ denotes an eigenvalue of $\mathbf{A}$ and $\boldsymbol{a}_{\mathrm{i}}$ is a vector determined by the corresponding eigenvector. From Eq. (26), it is clear that whether or not the solution or the flight is stable is decided by the eigenvalues $\lambda_{\mathrm{i}}(i=1-8) . \lambda_{\mathrm{i}}$ can be real or complex numbers (complex eigenvalues occur as conjugate pairs). Each real eigenvalue or each pair of complex eigenvalues gives a simple motion, and the solution is a linear combination of the simple motions. A simple motion is referred to as a natural mode of motion. If the one or several real eigenvalues are positive, or the real part of one or several complex eigenvalues are positive, the flight will be unstable.

\section{Results and Discussion}

The equilibrium flight. With the wing kinematics data at equilibrium flight given above, the aerodynamic forces at each flight speed can be computed. These are the aerodynamic forces at equilibrium flight. We use $x_{\mathrm{e}}^{+}$, $z_{\mathrm{e}}{ }^{+}$and $m_{\mathrm{e}}{ }^{+}$to represent the instantaneous non-dimensional forces and pitch moment at equilibrium flight. $x_{\mathrm{e}}{ }^{+}$is the horizontal force. $z_{\mathrm{e}}{ }^{+}$is the vertical force. $m_{\mathrm{e}}{ }_{\mathrm{e}}$ is the pitch moment. At equilibrium flight, because of the symmetry of the flow, only $x_{\mathrm{e}}^{+}, z_{\mathrm{e}}^{+}$and $m_{\mathrm{e}}{ }^{+}$are non-zero. The non-dimensional wingbeat-cycle averages of $x_{\mathrm{e}}^{+}, z_{\mathrm{e}}^{+}$ and $m_{\mathrm{e}}{ }^{+}$are $X_{\mathrm{e}}^{+}, Z_{\mathrm{e}}^{+}$and $M_{\mathrm{e}}{ }^{+}$, respectively. Subscript "w" denotes the contributions of the wing. Subscript "b" denotes the contributions of the body. Figure 4 shows the computed $x_{e, w}^{+}, x_{e, b}^{+}, z_{e, w}^{+}, z_{e, b}^{+}, m_{e, w}^{+}$and $m_{e, b}^{+}$in a flapping cycle at various flight speeds, after periodical state has been established.

The following observations can be made from Fig. 4. At hovering $\left(V_{\mathrm{e}}=0 \mathrm{~m} / \mathrm{s}\right.$, Fig. 4), positive vertical force (negative $z_{e, w}^{+}$) is produced in down- and upstrokes and $-z_{e, w}^{+}$in the downstroke is slightly larger than its counterpart in the upstroke. Both the down- and upstrokes contribute to the cycle-average force that supports the weight. Positive $x_{e, w}^{+}$is produced in the upstroke and negative $x_{e, w}^{+}$in the downstroke, and they have almost the same magnitude, resulting in an approximately zero mean horizontal force, as required for the hovering flight. At low speed $\left(V_{\mathrm{e}}=1.6 \mathrm{~m} / \mathrm{s}\right.$, Fig. 4$),-z_{e, w}^{+}$in the downstroke is much larger than its counterpart in the upstroke. Moreover, the downstroke duration is longer than that of the hovering case. Thus, the weight-supporting force is mainly generated by the downstroke. Positive $x_{e, w}^{+}$is still generated in the upstroke and negative $x_{e, w}^{+}$in the downstroke, but the magnitude of the negative $x_{e, w}^{+}$in the downstroke is smaller than that of the positive $x_{e, w}^{+}$in the upstroke, giving a positive cycle-average thrust. This shows that the force required for overcoming the drag of insect body is generated by the upstroke. At medium speed $\left(V_{\mathrm{e}}=3.1 \mathrm{~m} / \mathrm{s}\right.$, Fig. 4$),-z_{e, w}^{+}$is rather large during the downstroke but close to zero during the upstroke. Now the weight-supporting force is produced during the downstroke. As in the low-speed case, the force required for overcoming the drag of insect body is generated by the upstroke. At fast forward flight speeds $\left(V_{\mathrm{e}}=4.6-8.6 \mathrm{~m} / \mathrm{s}\right.$, Fig. 4$)$, as in the medium-speed case, $-z_{e, w}^{+}$is large in the downstroke and close to zero (even negative at $8.6 \mathrm{~m} / \mathrm{s}$ ) in the upstroke, and the downstroke duration is even longer. Again, the 

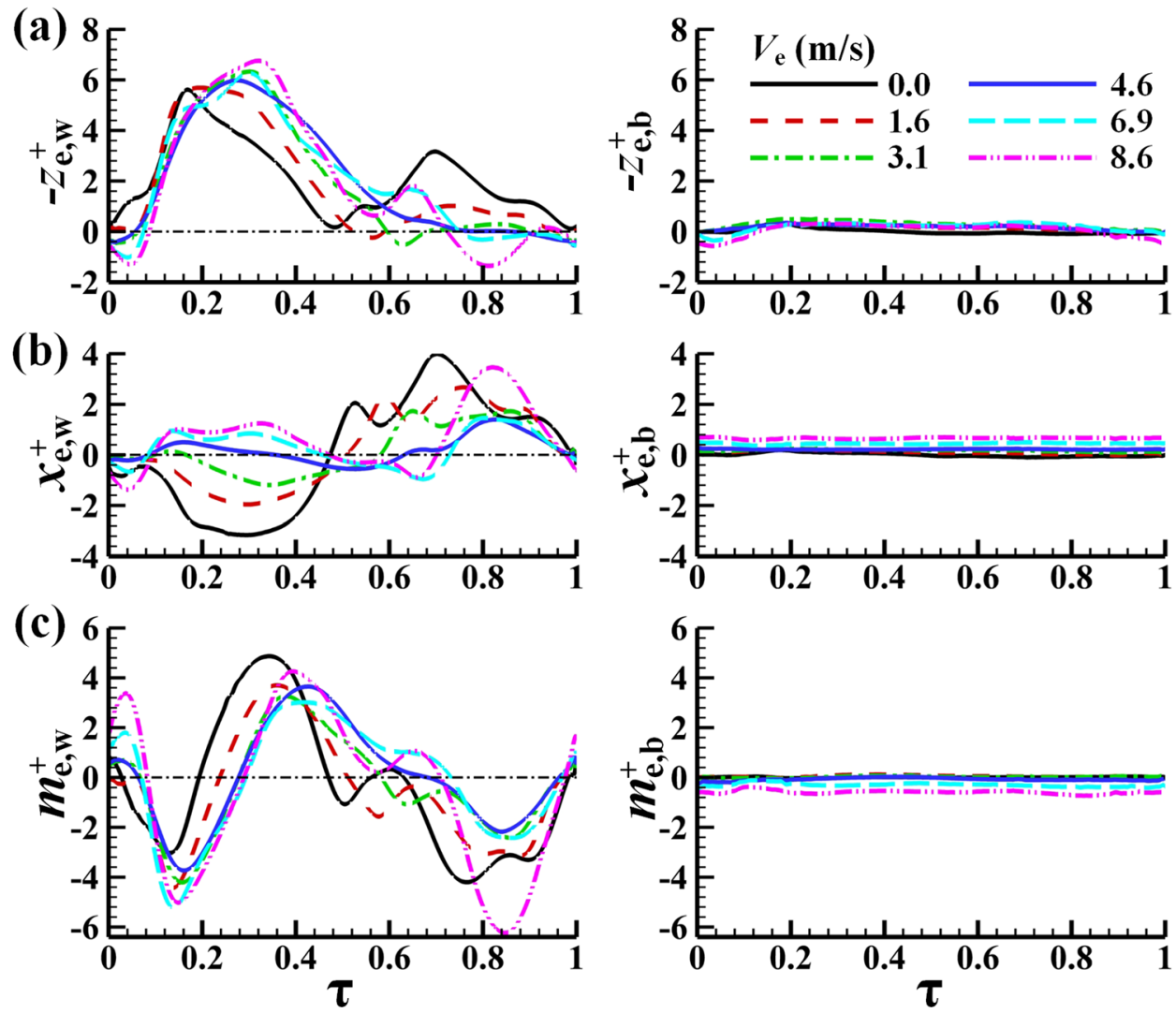

Figure 4. Time variations of the aerodynamic forces and moment of the wings and the body as functions of time in a flapping period (equilibrium flight).

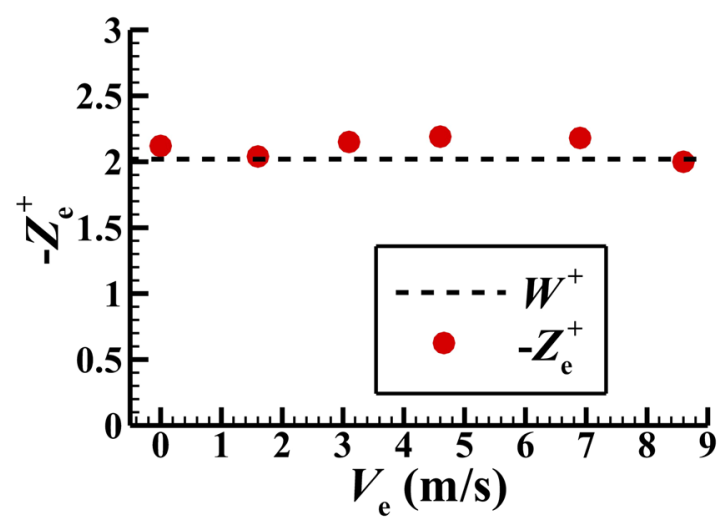

Figure 5. The calculated mean non-dimensional vertical force at various flight speeds, compared with the nondimensional weight.

weight-supporting force is produced in the downstroke. Unlike the medium-speed case, a large part of the downstroke ( $\tau=0.09-0.47$, Fig. 4 ) also produces positive $x_{e, w}^{+}$, showing that the thrust required for overcoming the body drag is produced by both the down- and upstrokes.

For constant-speed flight, the aerodynamic force in the vertical direction should approximately equal the insect-weight. The weight of the flies has been measured ${ }^{16}$ and the non-dimensionalized weight $\left(W^{+}\right)$is 2.02 . The computed non-dimensional vertical force of the wings plus that of the body, $-Z_{\mathrm{e}}^{+}$, for various flight speeds are shown in Fig. 5, compared with the non-dimensional weight $W^{+}$. At four flight speeds, the computed value is different from the measured by no more than $6 \%$; at $V_{\mathrm{e}}=4.6 \mathrm{~m} / \mathrm{s}$ and $6.9 \mathrm{~m} / \mathrm{s}$, the differences are about $8 \%$. We see that the requirement that the vertical force balances the weight is approximately satisfied.

The aerodynamic derivatives. To compute the aerodynamic derivatives with respect to a state variable, e.g. $u$, we vary the state variable $u$ from its equilibrium value (other state variables are kept unchanged) and calculate the aerodynamic forces, and moments, $X^{+}, Y^{+}, Z^{+} L^{+}, M^{+}$and $N^{+}$. As an example, the computed $X^{+}, Z^{+}$and 

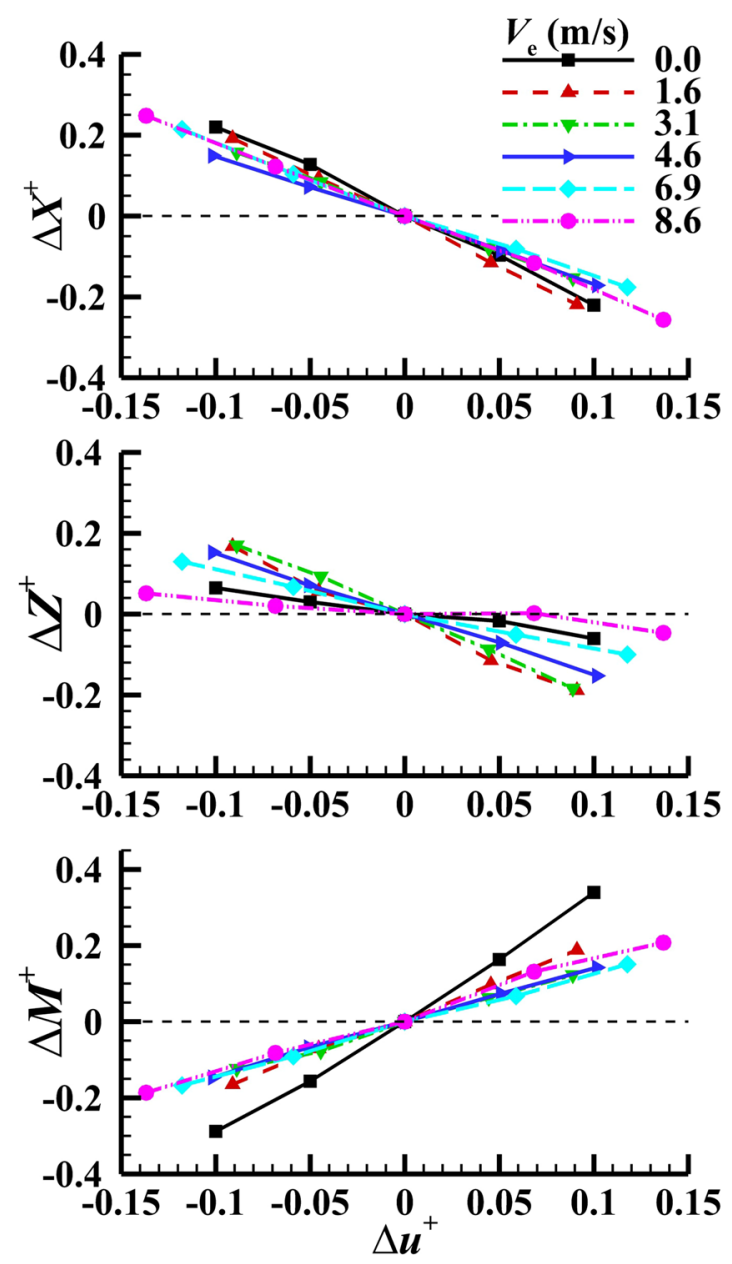

Figure 6. The $u$-series force and moment data.

$M^{+}$are shown in Fig. 6 (“ $\Delta^{\prime}$ " in the figure represents the difference between a variable and its equilibrium value). It is seen that $X^{+}, Z^{+}$and $M^{+}$vary with $u^{+}$approximately linearly when $-0.15 \leq \Delta u^{+} \leq 0.15$ (Fig. 6). This true for the cases of varying other state variables, justifying the linear theory for small perturbation motion. From the above results, aerodynamic derivatives can be computed. The computed aerodynamic derivatives, $X_{\mathrm{u}}^{+}, Z_{\mathrm{u}}^{+}, M_{\mathrm{u}}^{+}$, $X_{\mathrm{w}}{ }^{+}, Z_{\mathrm{w}}{ }^{+}, M_{\mathrm{w}}{ }^{+}, X_{\mathrm{q}}{ }^{+}, Z_{\mathrm{q}}{ }^{+}, M_{\mathrm{q}}{ }^{+}, X_{\mathrm{v}}{ }^{+}, Y_{\mathrm{v}}{ }^{+}, Z_{\mathrm{v}}{ }^{+}, L_{\mathrm{v}}{ }^{+}, M_{\mathrm{v}}{ }^{+}, N_{\mathrm{v}}{ }^{+}, X_{\mathrm{p}}{ }^{+}, Y_{\mathrm{p}}^{+}, Z_{\mathrm{p}}{ }^{+}, L_{\mathrm{p}}{ }^{+}, M_{\mathrm{p}}{ }^{+}, N_{\mathrm{p}}{ }^{+}, X_{\mathrm{r}}{ }^{+}, Y_{\mathrm{r}}^{+}, Z_{\mathrm{r}}{ }^{+}, L_{\mathrm{r}}{ }^{+}, M_{\mathrm{r}}{ }^{+}$and $N_{\mathrm{r}}{ }^{+}$are list in Table 3.

It is seen that the longitudinal derivatives due to the lateral motion are approximately 3 orders of magnitude smaller than other longitudinal derivatives (Table 3). As a result, the elements in the right and upper part of the system matrix $\mathbf{A}$ in Eq. (24) are approximately zero and $\mathbf{A}$ can be written as

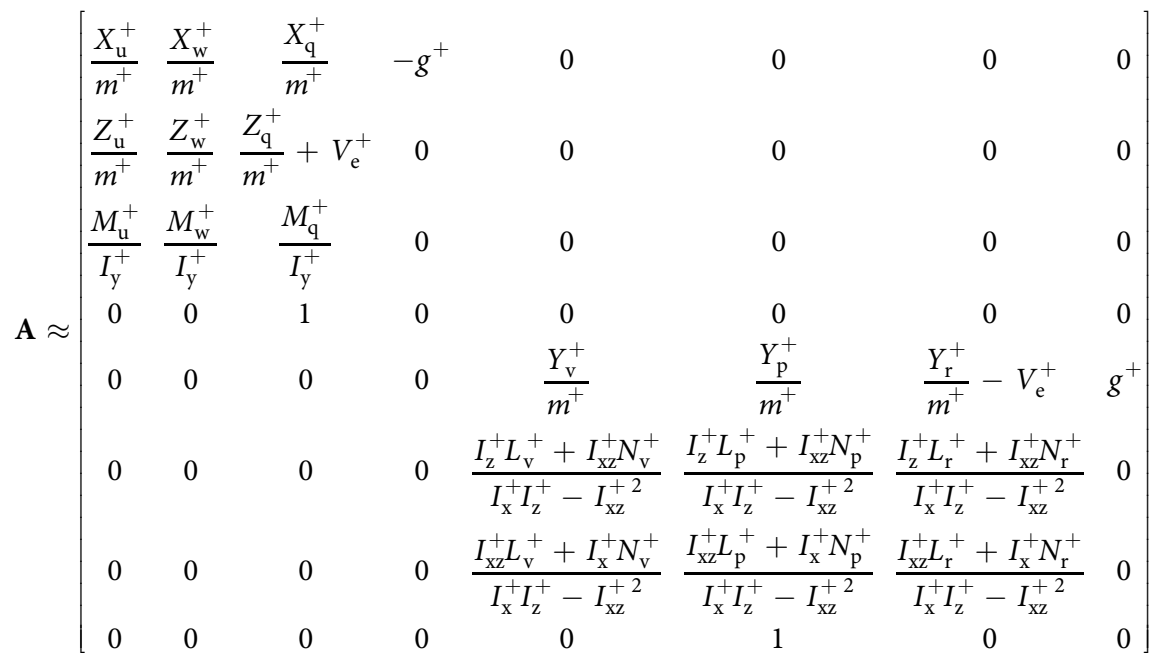




\begin{tabular}{|c|c|c|c|c|c|c|c|c|c|}
\hline \multicolumn{10}{|c|}{ Longitudinal derivatives } \\
\hline$V_{\mathrm{e}}(\mathrm{m} / \mathrm{s})$ & $X_{\mathrm{u}}^{+}$ & $Z_{\mathrm{u}}^{+}$ & $M_{\mathrm{u}}^{+}$ & $X_{\mathrm{w}}^{+}$ & $Z_{w}^{+}$ & $M_{w}{ }^{+}$ & $X_{\mathrm{q}}^{+}$ & $Z_{\mathrm{q}}^{+}$ & $M_{\mathrm{q}}^{+}$ \\
\hline 0.0 & -2.221 & -0.550 & 3.171 & 0.269 & -1.727 & -0.573 & -0.225 & -0.027 & -0.322 \\
\hline 1.6 & -2.272 & -1.917 & 1.892 & 0.107 & -2.638 & -0.277 & -0.131 & 0.041 & -0.368 \\
\hline 3.1 & -1.789 & -2.011 & 1.482 & -0.086 & -3.721 & -0.306 & -0.035 & -0.010 & -0.499 \\
\hline 4.6 & -1.564 & -1.453 & 1.408 & 0.240 & -4.585 & 0.611 & -0.065 & -0.043 & -0.571 \\
\hline 6.9 & -1.616 & -0.989 & 1.349 & 0.192 & -6.595 & 1.572 & 0.035 & -0.133 & -1.019 \\
\hline 8.6 & -1.794 & -0.245 & 1.638 & 0.431 & -7.224 & 2.698 & 0.092 & -0.228 & -1.305 \\
\hline \multicolumn{10}{|c|}{ Lateral derivatives } \\
\hline$V_{\mathrm{e}}(\mathrm{m} / \mathrm{s})$ & $Y_{\mathrm{v}}^{+}$ & $L_{\mathrm{v}}^{+}$ & $\mathrm{N}_{\mathrm{v}}^{+}$ & $\mathbf{Y}_{\mathrm{p}}^{+}$ & $L_{\mathrm{p}}^{+}$ & $\mathrm{N}_{\mathrm{p}}^{+}$ & $\boldsymbol{Y}_{\mathrm{r}}^{+}$ & $L_{\mathrm{r}}^{+}$ & $\mathrm{N}_{\mathrm{r}}^{+}$ \\
\hline 0.0 & -0.932 & -0.520 & 1.007 & -0.460 & -2.486 & -0.413 & 0.255 & 0.843 & -4.338 \\
\hline 1.6 & -1.145 & -1.070 & 2.081 & -0.278 & -2.765 & -0.009 & 0.432 & 1.816 & -2.913 \\
\hline 3.1 & -1.881 & -2.585 & 2.177 & -0.480 & -3.165 & 0.375 & 0.458 & 2.188 & -2.046 \\
\hline 4.6 & -1.998 & -2.486 & 1.140 & -0.694 & -4.653 & 0.340 & 0.388 & 2.770 & -2.005 \\
\hline 6.9 & -2.950 & -4.544 & 1.544 & -0.917 & -7.364 & 0.832 & 0.326 & 2.892 & -2.057 \\
\hline 8.6 & -3.981 & -6.006 & 1.342 & -1.086 & -9.675 & 0.904 & 0.552 & 2.937 & -2.491 \\
\hline \multicolumn{10}{|c|}{ Longitudinal derivatives due to lateral motion } \\
\hline$V_{\mathrm{e}}(\mathrm{m} / \mathrm{s})$ & $X_{\mathrm{v}}^{+}$ & $Z_{\mathrm{v}}^{+}$ & $M_{\mathrm{v}}^{+}$ & $X_{\mathrm{p}}^{+}$ & $Z_{\mathrm{p}}^{+}$ & $M_{\mathrm{p}}^{+}$ & $X_{\mathrm{r}}^{+}$ & $Z_{\mathrm{r}}^{+}$ & $M_{\mathrm{r}}^{+}$ \\
\hline 0.0 & \begin{tabular}{|l|}
0.004 \\
\end{tabular} & -0.012 & -0.005 & -0.001 & -0.003 & 0.003 & -0.003 & -0.002 & -0.001 \\
\hline 1.6 & 0.003 & -0.005 & 0.004 & 0.001 & 0.005 & -0.002 & -0.003 & -0.002 & 0.002 \\
\hline 3.1 & -0.004 & -0.020 & 0.001 & -0.002 & 0.002 & 0.001 & -0.002 & -0.006 & -0.002 \\
\hline 4.6 & -0.003 & -0.018 & 0.003 & -0.002 & -0.003 & 0.002 & 0.004 & 0.008 & 0.005 \\
\hline 6.9 & -0.002 & -0.001 & 0.001 & 0.000 & 0.006 & 0.003 & 0.008 & 0.004 & 0.000 \\
\hline 8.6 & \begin{tabular}{|l|}
0.000 \\
\end{tabular} & -0.007 & -0.012 & \begin{tabular}{|l|}
0.002 \\
\end{tabular} & 0.002 & 0.007 & 0.013 & 0.002 & -0.006 \\
\hline
\end{tabular}

Table 3. Aerodynamic derivatives.

Thus, as in the case of an airplane or helicopter, the linearization equations of motion of the insect can be separated into two equations, one giving longitudinal disturbance motion and the other giving lateral disturbance motion. The longitudinal one is:

$$
\left[\begin{array}{c}
\delta \dot{u}^{+} \\
\delta \dot{w}^{+} \\
\delta \dot{q}^{+} \\
\delta \dot{\theta}
\end{array}\right]=\mathbf{A}_{1}\left[\begin{array}{c}
\delta u^{+} \\
\delta w^{+} \\
\delta q^{+} \\
\delta \theta
\end{array}\right]
$$

where

$$
\mathbf{A}_{1}=\left[\begin{array}{cccc}
\frac{X_{\mathrm{u}}^{+}}{m^{+}} & \frac{X_{\mathrm{w}}^{+}}{m^{+}} & \frac{X_{\mathrm{q}}^{+}}{m^{+}} & -g^{+} \\
\frac{Z_{\mathrm{u}}^{+}}{m^{+}} & \frac{Z_{\mathrm{w}}^{+}}{m^{+}} & \frac{Z_{\mathrm{q}}^{+}}{m^{+}}+V_{\mathrm{e}}^{+} & 0 \\
\frac{M_{\mathrm{u}}^{+}}{I_{\mathrm{y}}^{+}} & \frac{M_{\mathrm{w}}^{+}}{I_{\mathrm{y}}^{+}} & \frac{M_{\mathrm{q}}^{+}}{I_{\mathrm{y}}^{+}} & 0 \\
0 & 0 & 1 & 0
\end{array}\right]
$$

and the lateral one is:

$$
\left[\begin{array}{c}
\delta \dot{v}^{+} \\
\delta \dot{p}^{+} \\
\delta \dot{r}^{+} \\
\delta \dot{\gamma}
\end{array}\right]=\mathbf{A}_{2}\left[\begin{array}{c}
\delta v^{+} \\
\delta p^{+} \\
\delta r^{+} \\
\delta \gamma
\end{array}\right]
$$

where 


\begin{tabular}{|l|l|l|l|l|}
\hline \multicolumn{5}{|l|}{ Longitudinal eigenvalues } \\
\hline $\boldsymbol{V}_{\mathbf{e}}(\mathbf{m} / \mathbf{s})$ & $\lambda_{\mathbf{l}}$ & $\boldsymbol{\lambda}_{\mathbf{2}}$ & $\boldsymbol{\lambda}_{3}$ & $\boldsymbol{\lambda}_{4}$ \\
\hline 0.0 & $0.0469+0.0967 \mathrm{i}$ & $0.0469-0.0967 \mathrm{i}$ & -0.1196 & -0.0139 \\
\hline 1.6 & $0.0307+0.0901 \mathrm{i}$ & $0.0307-0.0901 \mathrm{i}$ & -0.0864 & -0.0234 \\
\hline 3.1 & $0.0167+0.0955 \mathrm{i}$ & $0.0167-0.0955 \mathrm{i}$ & $-0.0452+0.0109 \mathrm{i}$ & $-0.0452-0.0109 \mathrm{i}$ \\
\hline 4.6 & 0.1007 & 0.0423 & -0.1833 & -0.0236 \\
\hline 6.9 & 0.2490 & 0.0142 & -0.3358 & -0.0205 \\
\hline 8.6 & 0.3803 & 0.0096 & -0.4765 & -0.0213 \\
\hline Lateral eigenvalues & \multicolumn{5}{l}{} \\
\hline $\boldsymbol{V}_{\mathbf{e}}(\mathbf{m} / \mathbf{s})$ & $\boldsymbol{\mu}_{1}$ & $\boldsymbol{\mu}_{2}$ & $\boldsymbol{\mu}_{3}$ & $\boldsymbol{\mu}_{4}$ \\
\hline 0.0 & 0.0478 & $-0.0779+0.0504 \mathrm{i}$ & $-0.0779-0.0504 \mathrm{i}$ & -0.5118 \\
\hline 1.6 & 0.0029 & $-0.0754+0.1622 \mathrm{i}$ & $-0.0754-0.1622 \mathrm{i}$ & -0.4926 \\
\hline 3.1 & -0.0012 & $-0.1129+0.2318 \mathrm{i}$ & $-0.1129-0.2318 \mathrm{i}$ & -0.4533 \\
\hline 4.6 & -0.0039 & $-0.0494+0.2261 \mathrm{i}$ & $-0.0494-0.2261 \mathrm{i}$ & -0.7811 \\
\hline 6.9 & -0.0044 & $-0.0403+0.2467 \mathrm{i}$ & $-0.0403-0.2467 \mathrm{i}$ & -1.2339 \\
\hline 8.6 & -0.0078 & $-0.0301+0.2417 \mathrm{i}$ & $-0.0301-0.2417 \mathrm{i}$ & -1.6376 \\
\hline
\end{tabular}

Table 4. Eigenvalues at various flight speeds.

$$
\mathbf{A}_{2}=\left[\begin{array}{cccc}
\frac{Y_{\mathrm{v}}^{+}}{m} & \frac{Y_{\mathrm{p}}^{+}}{m} & \frac{Y_{\mathrm{r}}^{+}}{m}-V_{\mathrm{e}}^{+} & g^{+} \\
\frac{I_{\mathrm{z}}^{+} L_{\mathrm{v}}^{+}+I_{\mathrm{xz}}^{+} N_{\mathrm{v}}^{+}}{I_{\mathrm{x}}^{+} I_{\mathrm{z}}^{+}-I_{\mathrm{xz}}^{+2}} & \frac{I_{\mathrm{z}}^{+} L_{\mathrm{p}}^{+}+I_{\mathrm{xz}}^{+} N_{\mathrm{p}}^{+}}{I_{\mathrm{x}}^{+} I_{\mathrm{z}}^{+}-I_{\mathrm{xz}}^{+2}} & \frac{I_{\mathrm{z}}^{+} L_{\mathrm{v}}^{+}+I_{\mathrm{xz}}^{+} N_{\mathrm{v}}^{+}}{I_{\mathrm{x}}^{+} I_{\mathrm{z}}^{+}-I_{\mathrm{xz}}^{+2}} & 0 \\
\frac{I_{\mathrm{xz}}^{+} L_{\mathrm{v}}^{+}+I_{\mathrm{z}}^{+} N_{\mathrm{v}}^{+}}{I_{\mathrm{x}}^{+} I_{\mathrm{z}}^{+}-I_{\mathrm{xz}}^{+2}} & \frac{I_{\mathrm{xz}}^{+} L_{\mathrm{p}}^{+}+I_{\mathrm{z}}^{+} N_{\mathrm{p}}^{+}}{I_{\mathrm{x}}^{+} I_{\mathrm{z}}^{+}-I_{\mathrm{xz}}^{+2}} & \frac{I_{\mathrm{xz}}^{+} L_{\mathrm{r}}^{+}+I_{\mathrm{z}}^{+} N_{\mathrm{r}}^{+}}{I_{\mathrm{x}}^{+} I_{\mathrm{z}}^{+}-I_{\mathrm{xz}}^{+2}} & 0 \\
0 & 0 & 1 & 0
\end{array}\right]
$$

$\mathbf{A}_{1}$ and $\mathbf{A}_{2}$ are the system matrixes of longitudinal motion and lateral motion, respectively.

From Table 3, one can see that several derivatives vary greatly as flight speed increasing. For example, $M_{\mathrm{w}}{ }^{+}$is negative at hovering $(-0.573)$, and slightly increases at $V_{\mathrm{e}}=1.6 \mathrm{~m} / \mathrm{s}$ and $3.1 \mathrm{~m} / \mathrm{s}(-0.277$ and -0.306$)$; however, when flight speed reaches $4.6 \mathrm{~m} / \mathrm{s}, M_{\mathrm{w}}{ }^{+}$becomes positive and then increases rapidly at high flight speeds $(0.611$, 1.572 and 2.698 at $V_{\mathrm{e}}=4.6 \mathrm{~m} / \mathrm{s}, 6.9 \mathrm{~m} / \mathrm{s}$ and $8.6 \mathrm{~m} / \mathrm{s}$, respectively). The above result indicates that the dynamic stability properties may change greatly at high flight speeds.

Flight stability. Now the derivatives $\left(X_{\mathrm{u}}^{+}, Z_{\mathrm{u}}{ }^{+}, M_{\mathrm{u}}^{+}\right.$, etc.) have been computed and matrices $\mathbf{A}_{1}$ and $\mathbf{A}_{2}$ of Eqs. (28)-(31) become known (parameters, $m^{+}, I_{\mathrm{x}}{ }^{+}$, etc., are calculated using the data in the section of Flight data). As discussed in the section of Solving the equations of motion, the eigenvalues of $\mathbf{A}_{1}$ and $\mathbf{A}_{2}$ give the stability properties of the dronefly. In the present paper, we use $\lambda$ and $\mu$ to denote the eigenvalues of the longitudinal and lateral system matrices. The results are given in Table 4.

First, we look at the case of hovering flight $\left(V_{\mathrm{e}}=0 \mathrm{~m} / \mathrm{s}\right)$. The results are similar to the previous results for dronefly $^{18}$ and bumblebee ${ }^{2}$. The longitudinal modes (Table $4, V_{\mathrm{e}}=0 \mathrm{~m} / \mathrm{s}$ ): an unstable slow oscillatory mode ( $\lambda_{1}$ and $\left.\lambda_{2}=0.0469 \pm 0.0967 i\right)$ and two stable subsidence modes, one fast $\left(\lambda_{3}=-0.1196\right)$ and the other slow $\left(\lambda_{4}=-0.0139\right)$. The lateral modes: an unstable slow divergence mode $\left(\mu_{1}=0.0478\right)$, a stable oscillatory mode $\left(\mu_{2}\right.$ and $\left.\mu_{3}=-0.0779 \pm 0.0504 i\right)$ and a stable fast subsidence mode $\left(\mu_{4}=-0.5118\right)$. Owing to the two unstable modes given by $\lambda_{1}$ and $\lambda_{2}$ and by $\mu_{1}$, hovering $\left(V_{\mathrm{e}}=0 \mathrm{~m} / \mathrm{s}\right)$ is not stable. But the instability is weak: the disturbance doubling time $\left(t_{\mathrm{d}}\right)$ is $83.0 \mathrm{~ms}$ for the longitudinal motion $\left(t_{\mathrm{d}}=0.693 /|\sigma|, \sigma\right.$ is the real part of the eigenvalue), about 15 wingbeat periods; for the lateral unstable mode, $t_{\mathrm{d}}=81.5 \mathrm{~ms}$, about the same as that of the longitudinal unstable mode.

Now we look at forward flight. First, we consider the longitudinal modes (Table 4). When flight speed is low $\left(V_{\mathrm{e}}=1.6 \mathrm{~m} / \mathrm{s}\right)$, the modal structure $\left(\lambda_{1}\right.$ and $\left.\lambda_{2}=0.0307 \pm 0.0901 i ; \lambda_{3}=-0.0864 ; \lambda_{4}=-0.0139\right)$ is same as that of hovering flight. At medium speed $\left(V_{\mathrm{e}}=3.1 \mathrm{~m} / \mathrm{s}\right)$, the modal structure changes to the following: two oscillatory modes, one being nearly neutral $\left(\lambda_{1}\right.$ and $\left.\lambda_{2}=0.0167 \pm 0.0955 i\right)$ and one being stable $\left(\lambda_{3}\right.$ and $\left.\lambda_{4}=-0.0452 \pm 0.0109 i\right)$. At higher speeds $\left(V_{\mathrm{e}}=4.6-8.6 \mathrm{~m} / \mathrm{s}\right)$, the modal structure changes again, having four real modes: the first one is a strongly unstable mode $\left(\lambda_{1}\right.$, positive and large), the second one is weakly unstable mode ( $\lambda_{2}$, positive and small), and the third and fourth ones are stable modes ( $\lambda_{3}$ and $\lambda_{4}$, negative). It is important to note that a large change in the stability property occurs when $V_{\mathrm{e}}$ is larger than about $V_{\mathrm{e}}=3.1 \mathrm{~m} / \mathrm{s}: \lambda_{1}$ increases greatly; that is, the instability become much stronger at high speeds. At $V_{\mathrm{e}}=8.6 \mathrm{~m} / \mathrm{s}, t_{\mathrm{d}}=10.1 \mathrm{~ms}$, approximately 8 times smaller than that of the hovering case.

Next we consider the lateral modes (Table 4). In the whole flight-speed range, modal structure does not change: two real modes and one oscillatory mode. The oscillatory mode $\left(\mu_{2}\right.$ and $\left.\mu_{3}\right)$ and one of the real mode (the fast one, $\mu_{4}$ ) are always stable. The mode represented by $\mu_{1}$ changes with speed as following. At hovering $\left(V_{\mathrm{e}}=0 \mathrm{~m} / \mathrm{s}\right)$ and low flight speed $\left(V_{\mathrm{e}}=1.6 \mathrm{~m} / \mathrm{s}\right)$, this mode is weakly unstable. At higher speeds $\left(V_{\mathrm{e}}=3.1-8.6 \mathrm{~m} / \mathrm{s}\right)$, 


\begin{tabular}{|l|l|l|l|l|}
\hline \multicolumn{5}{|l|}{ Longitudinal eigenvalues } \\
\hline $\boldsymbol{V}_{\mathbf{e}}(\mathbf{m} / \mathbf{s})$ & $\boldsymbol{\lambda}_{\mathbf{l}}$ & $\boldsymbol{\lambda}_{\mathbf{2}}$ & $\boldsymbol{\lambda}_{\mathbf{3}}$ & $\boldsymbol{\lambda}_{\mathbf{4}}$ \\
\hline 0.0 & $0.045+0.129 \mathrm{i}$ & $0.045-0.129 \mathrm{i}$ & -0.197 & -0.012 \\
\hline 1.0 & $0.060+0.102 \mathrm{i}$ & $0.060-0.102 \mathrm{i}$ & -0.172 & -0.023 \\
\hline 2.5 & $0.007+0.134 \mathrm{i}$ & $0.007-0.134 \mathrm{i}$ & $-0.048+0.044 \mathrm{i}$ & $-0.048-0.044 \mathrm{i}$ \\
\hline 3.5 & $0.012+0.120 \mathrm{i}$ & $0.012-0.120 \mathrm{i}$ & $0.074+0.042 \mathrm{i}$ & $0.074-0.042 \mathrm{i}$ \\
\hline 4.5 & 0.197 & 0.049 & -0.340 & -0.034 \\
\hline Lateral eigenvalues & \multicolumn{5}{|l|}{} \\
\hline $\boldsymbol{V}_{\mathbf{e}}(\mathbf{m} / \mathbf{s})$ & $\boldsymbol{\mu}_{1}$ & $\boldsymbol{\mu}_{2}$ & $\boldsymbol{\mu}_{3}$ & $\boldsymbol{\mu}_{4}$ \\
\hline 0.0 & 0.094 & $-0.118+0.072 \mathrm{i}$ & $-0.118-0.072 \mathrm{i}$ & -0.686 \\
\hline 1.0 & 0.045 & $-0.088+0.076 \mathrm{i}$ & $-0.088-0.076 \mathrm{i}$ & -0.914 \\
\hline 2.5 & 0.000 & $-0.119+0.208 \mathrm{i}$ & $-0.119-0.208 \mathrm{i}$ & -0.971 \\
\hline 3.5 & -0.006 & $-0.157+0.306 \mathrm{i}$ & $-0.157-0.306 \mathrm{i}$ & -1.088 \\
\hline 4.5 & -0.014 & $-0.161+0.266 \mathrm{i}$ & $-0.161-0.266 \mathrm{i}$ & -1.375 \\
\hline
\end{tabular}

Table 5. Eigenvalues of bumblebee at various flight speeds ${ }^{13,14}$.

this weakly unstable mode change to a very weakly stable one. The magnitude of $\mu_{1}$ keeps being very small thus this mode can be treated as a neutral mode. At all speeds, the two stable modes keep being stable, although the magnitude of $\mu_{4}$ increases greatly and that of the real part of $\mu_{2}$ (or $\mu_{3}$ ) decrease slightly.

The following can be stated from the above analysis. At $V_{\mathrm{e}}=0 \mathrm{~m} / \mathrm{s}$ (hover flight), the motion of the dronefly is weakly unstable owing to two weakly unstable natural modes of motion. At forward flight, at low and medium speeds $\left(V_{\mathrm{e}}=1.6 \mathrm{~m} / \mathrm{s}\right.$ and $\left.3.1 \mathrm{~m} / \mathrm{s}\right)$, when $V_{\mathrm{e}}$ increases, the stable modes tend to be more stable, and the unstable modes keep being weakly unstable or approximately neutral. However, at higher speeds $\left(V_{\mathrm{e}}=4.6-8.6 \mathrm{~m} / \mathrm{s}\right)$, the longitudinal unstable mode, represented by $\lambda_{1}$, becomes strongly unstable, $t_{\mathrm{d}}$ is $41.2,16.1$ and $10.1 \mathrm{~ms}$ for $V_{\mathrm{e}}=4.6 \mathrm{~m} / \mathrm{s}, 6.9 \mathrm{~m} / \mathrm{s}$ and $8.6 \mathrm{~m} / \mathrm{s}$, respectively.

Comparison with previous results. As discussed in the Introduction section, the flight stability in forward flight was studied previously using the flight data of a bumblebee. However, the flight data were only for $V_{\mathrm{e}}=0-4.5 \mathrm{~m} / \mathrm{s}^{15}$; data at higher flight speeds were not measured. The dronefly in the present study has approximately the same size and same wing kinematics as the bumblebee, and flight data for the whole speed range are available. Therefore, we will make a comparison of the present dronefly results with the previous results of the bumblebee.

The bumblebee's eigenvalues at various flight speed ${ }^{13,14}$ are listed in Table 5 . It is seen that the variations of the eigenvalue (i.e. the modes of motion) with the flight speed are similar to that of the dronefly. Especially, when $V_{\mathrm{e}}$ goes from $3.5 \mathrm{~m} / \mathrm{s}$ to $4.5 \mathrm{~m} / \mathrm{s}$, the longitudinal unstable mode $\left(\lambda_{1}\right)$ becomes much more unstable: at $V_{\mathrm{e}}=3.5 \mathrm{~m} / \mathrm{s}$ $t_{\mathrm{d}}=385 \mathrm{~ms}$, while at $V_{\mathrm{e}}=4.5 \mathrm{~m} / \mathrm{s} t_{\mathrm{d}}=23.5 \mathrm{~ms}$. With present dronefly data, it is shown that for higher flight speeds, the instability become even stronger at $V_{\mathrm{e}}=6.9 \mathrm{~m} / \mathrm{s}$ and $8.6 \mathrm{~m} / \mathrm{s}, t_{\mathrm{d}}$ become much smaller, $t_{\mathrm{d}}=16.1 \mathrm{~ms}$ and $10.1 \mathrm{~ms}$, respectively. We thus see that the present study extends the flight range of the previous study and provides the stability properties of the full speed range.

Possible effects of the strong instability at high flight speed. As seen above, at the highest flight speed $\left(V_{\mathrm{e}}=8.6 \mathrm{~m} / \mathrm{s}\right)$, the instability is very strong: the corresponding dimensional eigenvalue is $68.5 \mathrm{~s}^{-1}$ and $t_{\mathrm{d}}=10.1 \mathrm{~ms}$. The wingbeat period $T=5.6 \mathrm{~ms}(n=180 \mathrm{~Hz}$, Table 1$)$. That is, in less than two wingbeats, the magnitude of the disturbance will be doubled; this a very fast growth rate.

Some thoughtful experiments have been done by Ristroph et al. ${ }^{21}$ and they succeeded in measuring the control reaction time of fruit-flies. They showed that certain fast sensory organ can give control reaction in about 10-12 ms.

It is reasonable to assume that the dronefly has the same reaction delay time, approximately $11 \mathrm{~ms}$. We thus see that at the highest speed, the time for the disturbance to double its magnitude $\left(t_{\mathrm{d}}=10.1 \mathrm{~ms}\right)$ is approximately the same as the sensory reaction time of the fly. This indicates that the flight control is already difficult for flight at this speed. If flight speed is further increased, the instability may become even stronger; then the fly may not be able to control the flight. Therefore, we may say that strong flight instability may be one of the factors that limit the flight speed of a fly. Other factors could be very larger power requirement and inability to produce enough lift or thrust.

\section{Conclusions}

The longitudinal derivatives due to the lateral motion are approximately 3 orders of magnitude smaller than other longitudinal derivatives; thus, one can decouple the longitudinal motion from the lateral motion, as people do for a conventional airplane. At hovering flight, the motion of the dronefly is weakly unstable owing to two unstable natural modes of motion, a longitudinal one and a lateral one. At low $(1.6 \mathrm{~m} / \mathrm{s})$ and medium $(3.1 \mathrm{~m} / \mathrm{s})$ flight speeds, the unstable modes become even weaker and the flight is approximately neutral. At high flight speeds $(4.6 \mathrm{~m} / \mathrm{s}, 6.9 \mathrm{~m} / \mathrm{s}$ and $8.6 \mathrm{~m} / \mathrm{s})$, the flight becomes more and more unstable due to an unstable longitudinal mode. At the highest flight speed, $8.6 \mathrm{~m} / \mathrm{s}$, the instability is so strong that the time constant representing the growth rate of the instability (disturbance doubling time) is only $10.1 \mathrm{~ms}$, which is close to the sensory reaction time of a fly, approximately $11 \mathrm{~ms}$. This indicates that strong instability may play a role in limiting the flight speed of the insect. 
Received: 13 September 2019; Accepted: 17 January 2020;

Published online: 06 February 2020

\section{References}

1. Taylor, G. K. \& Thomas, A. L. R. Dynamic flight stability in the desert locust Schistocerca gregaria. J. Exp. Biol. 206, 2803-2829 (2003).

2. Sun, M. \& Xiong, Y. Dynamic flight stability of a hovering bumblebee. J. Exp. Biol. 208, 447-459 (2005).

3. Zbikowski, R., Ansari, S. A. \& Knowles, K. On mathematical modelling of insect flight dynamics in the context of micro air vehicles. Bioinspir. Biomim. 1, R26-R37 (2006).

4. Hedrick, T. L., Cheng, B. \& Deng, X. Y. Wingbeat Time and the Scaling of Passive Rotational Damping in Flapping Flight. Science (80-.). 324, 252-255 (2009).

5. Faruque, I. \& Humbert, J. S. Dipteran insect flight dynamics. Part 1 Longitudinal motion about hover. J. Theor. Biol. 264, 538-552 (2010).

6. Faruque, I. \& Humbert, J. S. Dipteran insect flight dynamics. Part 2: Lateral-directional motion about hover. J. Theor. Biol. 265, 306-313 (2010).

7. Liu, H. et al. Micro air vehicle-motivated computational biomechanics in bio-flights: aerodynamics, flight dynamics and maneuvering stability. Acta Mech. Sin. 26, 863-879 (2010).

8. Cheng, B. \& Deng, X. Y. Translational and rotational damping of flapping flight and its dynamics and stability at hovering. IEEE Trans. Robot. 27, 849-864 (2011).

9. Sun, M., Wang, J. K. \& Xiong, Y. Dynamic flight stability of hovering insects. Acta Mech. Sin. 23, 231-246 (2007).

10. Xu, N. \& Sun, M. Lateral flight stability of two hovering model insects. J. Bionic Eng. 11, 439-448 (2014).

11. Liu, L. G. \& Sun, M. Dynamic flight stability of hovering mosquitoes. J. Theor. Biol. 464, 149-158 (2019).

12. Sun, M. Insect flight dynamics: Stability and control. Rev. Mod. Phys. 86, 615-646 (2014).

13. Xiong, Y. \& Sun, M. Dynamic flight stability of a bumblebee in forward flight. Acta Mech. Sin. 24, 25-36 (2008)

14. Xu, N. \& Sun, M. Lateral dynamic flight stability of a model bumblebee in hovering and forward flight. J. Theor. Biol. 319, 102-115 (2013).

15. Dudley, R. \& Ellington, C. P. Mechanics of forward flight in bumblebees. I. Kinematics and morphology. J. Exp. Biol. 148, 19-52 (1990).

16. Meng, X. G. \& Sun, M. Wing and body kinematics of forward flight in drone-flies. Bioinspir. Biomim. 11, 56002 (2016).

17. Etkin, B. \& Reid, L. D. Dynamics of flight: stability and control. (John Wiley \& Sons, Inc., 1996).

18. Zhang, Y. L. \& Sun, M. Dynamic flight stability of a hovering model insect: lateral motion. Acta Mech. Sin. 26, 175-190 (2010).

19. Sun, M. \& Tang, J. Unsteady aerodynamic force generation by a model fruit fly wing in flapping motion. J. Exp. Biol. 205, 55-70 (2002).

20. Cheng, X. \& Sun, M. Very small insects use novel wing flapping and drag principle to generate the weight-supporting vertical force. J. Fluid Mech. 855, 646-670 (2018).

21. Ristroph, L. et al. Discovering the flight autostabilizer of fruit flies by inducing aerial stumbles. Proc. Natl. Acad. Sci. USA 107, 4820-4824 (2010).

\section{Acknowledgements}

This research was supported by grants from the National Natural Science Foundation of China (11832004 and 11721202).

\section{Author contributions}

Experiments were planned by X.G.M. and M.S. and conducted by X.G.M and H.J.Z. The results were analysed by all authors. The manuscript was written by M.S. and reviewed by all authors.

\section{Competing interests}

The authors declare no competing interests.

\section{Additional information}

Supplementary information is available for this paper at https://doi.org/10.1038/s41598-020-58762-5.

Correspondence and requests for materials should be addressed to H.J.Z.

Reprints and permissions information is available at www.nature.com/reprints.

Publisher's note Springer Nature remains neutral with regard to jurisdictional claims in published maps and institutional affiliations.

(c) (i) Open Access This article is licensed under a Creative Commons Attribution 4.0 International License, which permits use, sharing, adaptation, distribution and reproduction in any medium or format, as long as you give appropriate credit to the original author(s) and the source, provide a link to the Creative Commons license, and indicate if changes were made. The images or other third party material in this article are included in the article's Creative Commons license, unless indicated otherwise in a credit line to the material. If material is not included in the article's Creative Commons license and your intended use is not permitted by statutory regulation or exceeds the permitted use, you will need to obtain permission directly from the copyright holder. To view a copy of this license, visit http://creativecommons.org/licenses/by/4.0/.

(C) The Author(s) 2020 